\title{
Calibration and cross-laboratory implementation of scintillating screens for electron bunch charge determination
}

Thomas Kurz, Jurjen Pieter Couperus, Jakob Matthias Krämer, Hao Ding, Stephan Kuschel, Alexander Köhler, Omid Zarini, Dominik Hollatz, David Schinkel, Richard D’Arcy, Jan-Patrick Schwinkendorf, Jens Osterhoff, Arie Irman, Ulrich Schramm, and Stefan Karsch

Citation: Review of Scientific Instruments 89, 093303 (2018); doi: 10.1063/1.5041755

View online: https://doi.org/10.1063/1.5041755

View Table of Contents: http://aip.scitation.org/toc/rsi/89/9

Published by the American Institute of Physics

\section{Articles you may be interested in}

Absolute charge calibration of scintillating screens for relativistic electron detection

Review of Scientific Instruments 81, 033301 (2010); 10.1063/1.3310275

Chemical-vapor deposited ultra-fast diamond detectors for temporal measurements of ion bunches

Review of Scientific Instruments 89, 093304 (2018); 10.1063/1.5048667

An angular-resolved multi-channel Thomson parabola spectrometer for laser-driven ion measurement Review of Scientific Instruments 89, 093302 (2018); 10.1063/1.5042424

Absolute calibration for a broad range single shot electron spectrometer Review of Scientific Instruments 77, 103301 (2006); 10.1063/1.2360988

Control of quasi-monoenergetic electron beams from laser-plasma accelerators with adjustable shock density profile

Physics of Plasmas 25, 043107 (2018); 10.1063/1.5023694

Novel scintillator-based $x$-ray spectrometer for use on high repetition laser plasma interaction experiments Review of Scientific Instruments 89, 073502 (2018); 10.1063/1.5019213

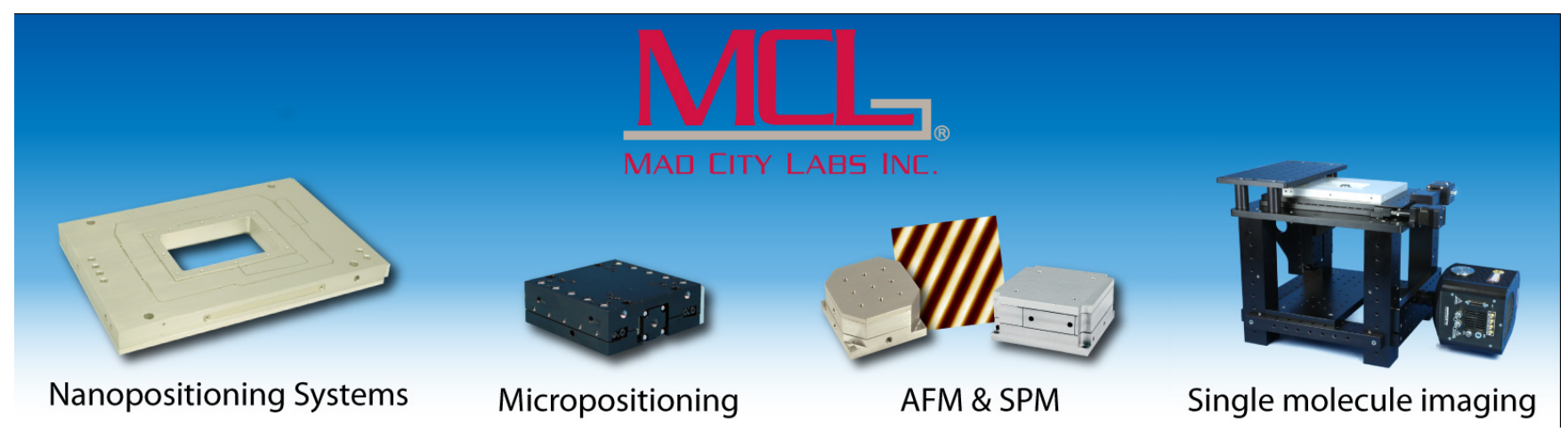




\title{
Calibration and cross-laboratory implementation of scintillating screens for electron bunch charge determination
}

\author{
Thomas Kurz, ${ }^{1,2,3, a)}$ Jurjen Pieter Couperus, ${ }^{1,3}$ Jakob Matthias Krämer, ${ }^{1,3}$ Hao Ding, ${ }^{2,4}$ \\ Stephan Kuschel, ${ }^{5,6}$ Alexander Köhler, ${ }^{1,3}$ Omid Zarini, ${ }^{1,3}$ Dominik Hollatz, ,,6 \\ David Schinkel, ${ }^{5,6}$ Richard D'Arcy, ${ }^{7}$ Jan-Patrick Schwinkendorf, ${ }^{7,8}$ Jens Osterhoff, ${ }^{7}$ \\ Arie Irman, ${ }^{1}$ Ulrich Schramm, ${ }^{1,3}$ and Stefan Karsch ${ }^{2,4}$ \\ ${ }^{1}$ Helmholtz-Zentrum Dresden-Rossendorf, Bautzner Landstraße 400, 01328 Dresden, Germany \\ ${ }^{2}$ Ludwig-Maximilians-Universität München, Am Coulombwall 1, 85748 Garching, Germany \\ ${ }^{3}$ Technische Universität Dresden, 01069 Dresden, Germany \\ ${ }^{4}$ Max-Planck-Institut für Quantenoptik, Hans-Kopfermann-Straße 1, 85748 Garching, Germany \\ ${ }^{5}$ Helmholtz-Institut Jena, Fröbelstieg 3, 07743 Jena, Germany \\ ${ }^{6}$ Friedrich-Schiller-Universität Jena, Fürstengraben 1, 07743 Jena, Germany \\ ${ }^{7}$ Deutsches Elektronen-Synchrotron DESY, Notkestraße 85, 22607 Hamburg, Germany \\ ${ }^{8}$ Institut für Experimentalphysik, Universität Hamburg, 22761 Hamburg, Germany
}

(Received 25 May 2018; accepted 27 August 2018; published online 20 September 2018)

\begin{abstract}
We revise the calibration of scintillating screens commonly used to detect relativistic electron beams with low average current, e.g., from laser-plasma accelerators, based on new and expanded measurements that include higher charge density and different types of screens than previous work [Buck et al., Rev. Sci. Instrum. 81, 033301 (2010)]. Electron peak charge densities up to $10 \mathrm{nC} / \mathrm{mm}^{2}$ were provided by focused picosecond-long electron beams delivered by the Electron Linac for beams with high Brilliance and low Emittance (ELBE) at the Helmholtz-Zentrum Dresden-Rossendorf. At low charge densities, a linear scintillation response was found, followed by the onset of saturation in the range of $\mathrm{nC} / \mathrm{mm}^{2}$. The absolute calibration factor (photons/sr/pC) in this linear regime was measured to be almost a factor of 2 lower than that reported by Buck et al. retrospectively implying a higher charge in the charge measurements performed with the former calibration. A good agreement was found with the results provided by Glinec et al. [Rev. Sci. Instrum. 77, 103301 (2006)]. Furthermore long-term irradiation tests with an integrated dose of approximately $50 \mathrm{nC} / \mathrm{mm}^{2}$ indicate a significant decrease of the scintillation efficiency over time. Finally, in order to enable the transfer of the absolute calibration between laboratories, a new constant reference light source has been developed. Published by AIP Publishing. https://doi.org/10.1063/1.5041755
\end{abstract}

\section{INTRODUCTION}

Triggered by the proposal of Tajima and Dawson ${ }^{1}$ in 1979, the development of laser-plasma accelerators (LPAs) has progressed tremendously. Continuous advancement in both the understanding and control over the electron injection and acceleration mechanisms ${ }^{2,3}$ and the development of state of the art laser systems up to the petawatt regime $e^{4,5}$ enables the acceleration of quasi-monoenergetic ${ }^{6-8}$ electron bunches to energies in the $\mathrm{GeV}$ range ${ }^{9-11}$ and peak charges in the $\mathrm{nC}$ range ${ }^{12}$ within only $\mathrm{cm}$-long acceleration distance. Compared to electron beams from conventional RF accelerators, LPA beams possess unique characteristics, such as ultra-short duration and high peak current, ${ }^{13}$ but still suffer from shotto-shot fluctuations. Moreover the high intensity laser-plasma interaction creates a harsh environment where most of the conventional diagnostics fail. Therefore an appropriate diagnostic method is demanding. In particular, for the measurement of energy-resolved single shot electron bunch charge, a method capable of covering a broad parameter range is required. Typical bunches contain a charge of a few $\mathrm{pC}$ up to $\mathrm{nC}$ within ps-to-fs duration with an energy distribution ranging from

a)E-mail: t.kurz@hzdr.de
$10 \mathrm{MeV}$ to the $\mathrm{GeV}$ level. Typically this is measured by a permanent or current-driven dipole magnet of $\mathrm{m}$-scale length, which maps the electron energy to the position in the dispersive plane, in combination with a correspondingly large (few hundreds of $\mathrm{cm}^{2}$ ) scintillating screen imaged onto a CCD camera for charge diagnostic. ${ }^{5}$ The majority of scintillation screens used in this work consist of a $10 \mu \mathrm{m}-100 \mu \mathrm{m}$-thick layer of powdered rare earth phosphor $(\mathrm{Gd} 2 \mathrm{O} 2 \mathrm{~S}: \mathrm{Tb})$, which converts a fraction of the deposited electron energy into visible light. This process is dominated by fluorescence and has a lifetime of approximately $1 \mathrm{~ms}^{14}$ The short life-time enables single-shot diagnostic at repetition rates of up to $1 \mathrm{kHz}$. By contrast, imaging plates, ${ }^{15-18}$ which deliver a good spatial resolution and high dynamic range, suffer from a long offline readout procedure. Scintillating screens are commercially available, often under the trade name LANEX, and marketed for X-ray detection. They are manufactured in various spatial resolutions and sensitivities and are supplied by several companies. Thus, the choice of screen depends on the specific experimental requirements. Aimed for x-ray detection, generally no electron-photon conversion efficiency is specified, and therefore, careful calibration is required in order to extract quantitative electron charge density data from screen images. 
For this reason, calibration studies of scintillators have been performed some years ago by Glinec et al. ${ }^{19}$ Buck et al. ${ }^{20}$ and Nakamura et al. ${ }^{21}$ Among these, the work of Buck et al. ${ }^{20}$ performed by the consortium authoring this work provides an absolute and transportable charge calibration for various scintillating screens, allowing different laboratories to use this calibration to compare the obtained electron bunch charge across different experiments. Here, we update and extend this work toward higher charge densities, which reflects recent improvements especially in LPAs. In this new parameter range, more emphasis has to be given to saturation and radiation damage effects. Additionally, we update the range of screen types according to current commercial availability. Thus, the setup (Sec. II) has been revised compared to the earlier work, ${ }^{20}$ charge densities have been adjusted to better meet the conditions of modern high-charge LPAs, and the range of the linear response (Sec. III A) as well as the saturation (Sec. III B) of the scintillating screens has been investigated. Previously unavailable information on the long-term stability and damage resistance under repeated high-flux irradiation is reported in Sec. III C. In Sec. IV, a new concept for the cross-calibration of the scintillating screens is described. This concept enables to implement the calibration results in other laboratories. This study is intended to replace the previous study by Buck et al. ${ }^{20}$

\section{EXPERIMENTAL SETUP}

The setup for the absolute charge calibration of the scintillation screens is illustrated in Fig. 1. The measurements were performed at the Electron Linac for beams with high Brilliance and low Emittance (ELBE) linear accelerator (LINAC) at the Helmholtz-Zentrum Dresden-Rossendorf. For this campaign, the sub-10 ps long electron bunches with a charge of $15 \mathrm{pC}$ at an energy of $23.5 \mathrm{MeV}$ were provided by the LINAC. In order to extend the calibration range to higher charges, the accelerator can be operated in a bunch train mode with multiple pulses in one train of tunable length running at a repetition rate of $13 \mathrm{MHz}$. The temporal spacing between the pulses corresponds to $77 \mathrm{~ns}$. Since the lifetime of the excited state of the scintillator is $\sim 1 \mathrm{~ms},{ }^{14}$ the charge of $15 \mathrm{pC}$ contained in each micro-bunch in a train of up to 1400 bunches adds up which in total forms a macro-bunch with a charge of up to $21 \mathrm{nC}$. After focusing the electron beam by magnetic quadrupoles to a full width at half maximum (FWHM) area of $2 \mathrm{~mm}^{2}-3 \mathrm{~mm}^{2}$, this corresponds to a peak charge density of up to $10 \mathrm{nC} / \mathrm{mm}^{2}$. Although measurements are performed at a fixed electron energy of $23.5 \mathrm{MeV}$, the calibration applies reliably over a broad energy range. Simulations show that the energy deposition of the electrons inside the photo-luminescent layer is almost independent of their kinetic energy above a threshold value of $3 \mathrm{MeV} \cdot{ }^{16,19,22,23}$ Additionally, Nakamura et al. ${ }^{21}$ demonstrated that the sensitivity of Lanex Fast to highly relativistic electrons has only a weak dependency of the electron energy. It decreases by $1 \%$ per $100 \mathrm{MeV}$ increase of the electron energy. Thus the calibration results can be used to determine the charge of relativistic electron bunches produced by LPAs.

Directly before interacting with the screen, the charge of each electron micro-bunch is measured by an integrating current transformer (ICT-082-070-05:1-VAC, Bergoz Instrumentation, France). The ICT pulses were amplified by a factor of 56 (Pulse Amplifier Coaxial ZPUL-30P, Mini Circuits, USA) and recorded by a high resolution oscilloscope ( $2 \mathrm{GHz}$ RTE 1204, Rhode \& Schwarz, Germany). The fluorescence emission from the scintillating screens exhibits an angular distribution following approximately the Lambertian law. ${ }^{23,24}$ The setup geometry was chosen such that the camera collects light in the direction normal to the screen which maximizes the light detected by the CCD chip. The scintillating screens were placed within a vacuum vessel which was directly connected to the main accelerator. Furthermore a metallic turning mirror (PF20-03-P01, Thorlabs) was mounted off-axis to avoid generating background optical transition radiation (OTR) that would otherwise contribute to the signal detected on the CCD chip. These precautions improved on the setup used in the original work of Buck et al. ${ }^{17,20}$ The screens were mounted on a rotating target wheel which was aligned $(22 \pm 1)^{\circ}$ relative to the electron beam. The emitted photons with a peak wavelength $\lambda_{\text {peak }}$ of $546 \mathrm{~nm}$ are reflected by the mirror to a 12-bit CCD camera (acA1300-30gm, Basler) equipped with a teleobjective (Ricoh FL-CC6Z1218A-VG, Ricoh, Japan) having a focal length of $(53 \pm 2) \mathrm{mm}$ and an $\mathrm{f}$-number of 1.8 . For the broad charge range treated in this work, a detection system with a dynamic range of three orders of magnitude is required. This is achieved by a filter wheel equipped with ND filters ranging from ND0.5 to ND4.0 which were calibrated precisely (below

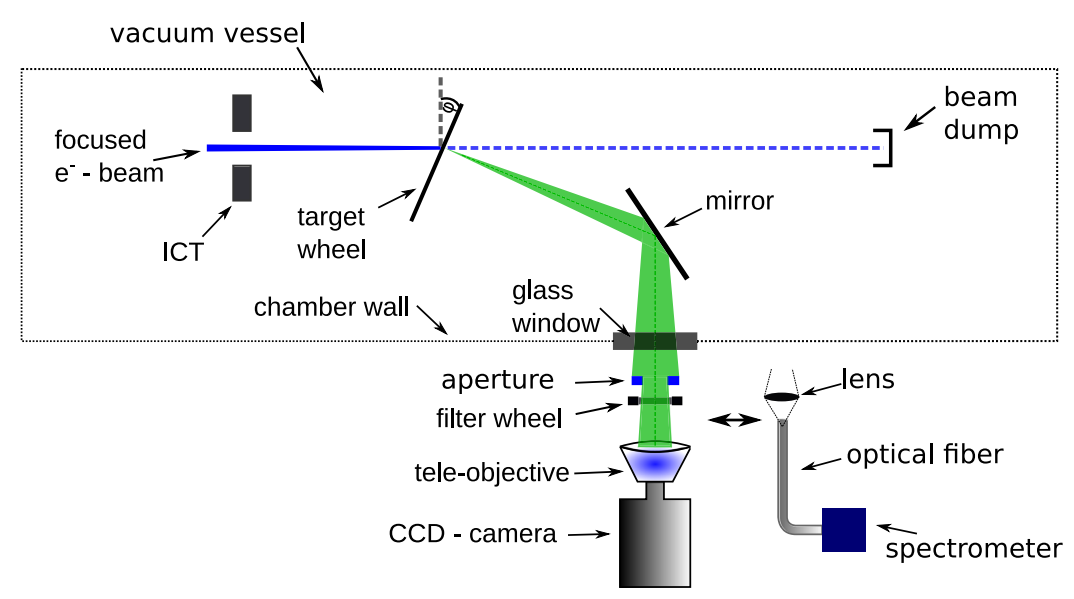

FIG. 1. Setup for absolute charge calibration of scintillation screens: The ICT measures the charge of the electron beam. Six different screens with an angle of $22^{\circ}$ relative to the incoming electron beam were mounted on a rotating target wheel and optically imaged via a silver mirror onto a CCD chip. In order to generate three orders of magnitude of dynamic range, a set of ND filters was placed in front of the camera. The effective collection angle is defined by an aperture in front of the telephoto lens. 
$0.5 \%$ uncertainty) using a well-calibrated spectrophotometer (Cary 50 UV-VIS, Agilent Technologies). This approach guarantees a more precise measurement of the scintillator's brightness (photons/sr) over the entire calibration curve, as compared to that of Buck et al. ${ }^{20}$ where an adjustable iris was used. Additionally an optical fiber (M200L02S-A, Thorlabs) connected to a spectrometer (HR4000, Ocean Optics) was used to determine the fluorescence spectrum of the scintillation screens. This information is required to determine the camera efficiency of the CCD and the effective attenuation of the ND filters over the whole scintillation spectrum. In order to cross-reference the scintillation signal with a master light source, a tritium source was added to a free position on the filter wheel. The solid angle in our optical detection system was $(3.18 \pm 0.07) \times 10^{-3}$ sr defined by an aperture diameter of $(22.96 \pm 0.05) \mathrm{mm}$ mounted at a distance of $(361 \pm 4) \mathrm{mm}$ from the target. For this small solid angle, the lateral signal variation due to the Lambertian emission characteristic can be ignored.

\section{RESULTS}

\section{A. Absolute charge calibration}

The absolute calibration (total photons/sr/pC) of the scintillation screens serves as a universal reference for charge diagnostics with particular importance on the field of LPAs. Therefore the transmission efficiency of the optical detection system was measured to determine the brightness (photons/sr) of the scintillator. Together with a precise knowledge (5\% uncertainty) of the LINAC's bunch charge, the absolute scintillation efficiency can be determined.

A representative electron beam image is shown in Fig. 2. The brightness (photons) of the scintillating screens is measured as the integrated CCD counts within the region of interest (ROI) and corrected for the background originating from the

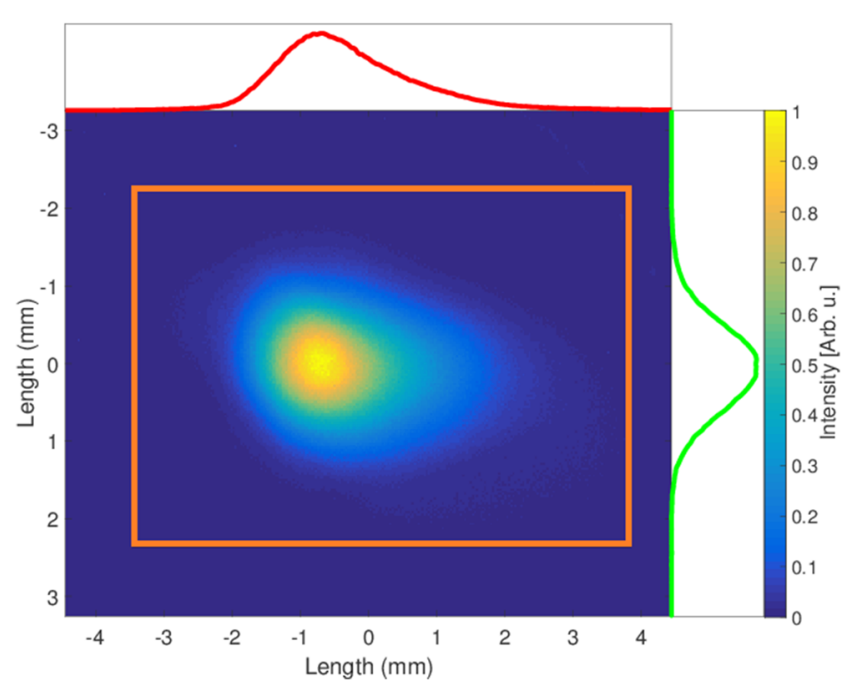

FIG. 2. Image of an electron bunch recorded by the CCD sensor. The rectangle marks the region of interest (ROI) which was used for the analysis. The two curves indicate the line-out of the electron bunch through its peak in horizontal and vertical directions. The area of the bunch at FWHM is $\approx 2 \mathrm{~mm}^{2}$. camera, i.e., thermal noise, the accelerator's dark current, and bremsstrahlung. Accordingly, the absolute response of the scintillating screen $C_{\mathrm{S}}$, i.e., the total number of photons $N_{\mathrm{ph}}$ emitted by the scintillator into an area of one steradian per incident electron charge $Q_{\mathrm{e}}$ is given by

$$
C_{\mathrm{S}}=\frac{N_{\mathrm{ph}}}{Q_{\mathrm{e}}}=\frac{N_{\mathrm{c}} \cos (\varphi)}{\beta \Omega Q_{\mathrm{e}}},
$$

where $N_{\mathrm{c}}$ describes the total number of counts within the ROI of the background-corrected image. $\varphi$ is the angle between the electron beam and the normal vector of the scintillator's surface. The cosine corrects the photon signal recorded by the CCD camera for the increased interaction length due to the incidence angle of the electrons. $\Omega$ symbolizes the effective collection angle in units of steradian. Furthermore, $\beta$ denotes the light collection efficiency of the optical detection system. For the sake of completeness, $\beta$ can be disassembled in its individual contributions. The reflection of the off-axis mirror at the specific wavelength is $(97 \pm 1) \%$, the window of the vacuum chamber transmits $(91.3 \pm 0.5) \%$ of the incoming light, and the objective transmits $(88 \pm 1) \%$ of the photons to the chip. The photon-to-count conversion efficiency of $(32.8$ $\pm 1.7) \%$ of the CCD chip (ICX445, Sony) and its associated readout-electronics were determined separately using a green laser and a reference power detector (XLP12-3SH2-D0, Gentec International, Canada).

The response functions for the different screens as a function of the bunch charge are shown in Fig. 3. All curves show a linear behavior up to a threshold caused by saturation and degeneration effects (Secs. III B and III C). In order to determine the calibration value for the absolute response of the different scintillators, a linear fit has been applied to all data points that deviate less than $-10 \%$ from linearity as indicated by the filled markers. As the upper limit of this linear region depends on the areal charge density, it obviously depends on the beam size (see Sec. III B). The resulting calibration values are shown in the second column of Table I. It should be noted that only a single sample of each type of screen was investigated systematically. Potential batch-to-batch variations have not been investigated. ${ }^{27}$

The overall uncertainty of the absolute fluorescence efficiency of $16 \%$ (see Table I) originates from statistical and systematic uncertainties. The standard error of the mean (average over 50 shots) of the raw images together with the error of the linear fit adds up to approximately $2 \%$. The main contribution is due to systematic uncertainties. The error in the determination of the bunch charge $Q_{\mathrm{e}}$ was determined to be $5 \%$, originating from the systematic error of the ICT measurement $^{20}$ and the uncertainty of the analysis algorithm. An error of $6 \%$ is accounted to the uncertainty of the transmission efficiency $\beta$, which includes optics in the beamline (see Fig. 1) as well as the photon-to-count efficiency of the CCD-camera. Furthermore, the cosine error caused by the deviation of the alignment angle $\varphi$ and the solid angle $\Omega$ (see Sec. II) adds up to the remaining uncertainty of $3 \%$. A detailed overview of all the relevant quantities for the determination of the absolute fluorescence efficiency can be found in Table II.

When comparing the absolute calibration results to those of Buck et al. ${ }^{20}$ the sensitivity ratios between the screens 


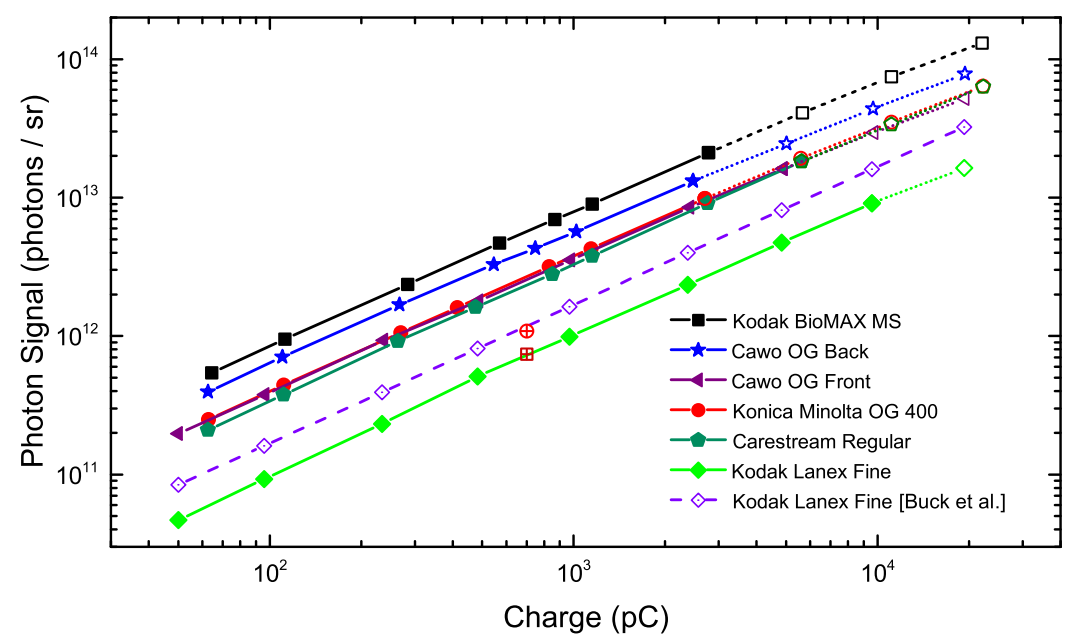

FIG. 3. Absolute charge calibration of six different scintillation screens. The linearity hypothesis is valid up to a certain charge density threshold where the fluorescence signal is significantly lowered. We define the threshold at a drop in signal of more than $10 \%$ below the low-charge linear fit. Beyond this threshold, nonlinear saturation effects and beam induced damage effects start to play a role in the photon response. The solid line of each calibration curve indicates the linear region, whereas the dotted line shows the non-linear part. The dashed line displays the calibration curve for Kodak Lanex Fine from Buck et al. ${ }^{20}$ Additionally two reference data points for Kodak Lanex Fine are included. The red circle is determined by a calculation based on a Monte Carlo simulation reported in the work of Glinec et al. ${ }^{19}$ as referenced in the work of Buck et al. The red square was deduced from the full set of experimental results given by Glinec et al. (see the supplementary material). The FWHM area of the beam is $\sim 2 \mathrm{~mm}^{2}$. agree quite well. However, a constant reduction of the overall sensitivity by roughly a factor of two is found in this work. The deviation might originate from two shortcomings in the setup of the previous work ${ }^{20}$ which offer potential interfering background sources. First, in order to image the scintillating screen, a mirror was placed on the beam axis. As a source of OTR light, this mirror can add a significant amount of extra signal as partially collected by the camera. ${ }^{25}$ Second, the electron beam was coupled out from the vacuum chamber via a $100 \mu$ m-thick beryllium window, potentially causing an extra charge-dependent X-ray background which the scintillating screen converts into light. The latter will add only a small amount of background signal; nevertheless, in the current calibration setup, such an X-ray contribution was avoided.
The deviation of the absolute scintillation responses compared to those published by Buck et al. ${ }^{20}$ prompted us to repeat the experiment in three independent campaigns in order to exclude sources of systematic errors. The setup for each campaign was identical apart from small $(10 \%)$ variations in the collection angle, which have been taken into account in the analysis. The comparison of the absolute scintillating signal of each screen measured in the different calibration campaigns leads to similar $( \pm 5 \%)$ results increasing the confidentiality of the obtained calibration values. Additionally, it was found that the scintillation efficiency based on the experimental values published by Glinec et al. ${ }^{19}$ shows good agreement to the current value. The data provided by Glinec lead to an absolute conversion efficiency for KODAK

TABLE I. Calibration values for different scintillation screens: results for the absolute fluorescence efficiency (second column) and the saturation threshold (third column) as well as the resulting fit parameter (fourth column). The presented values reflect the absolute fluorescence efficiency and saturation behavior of fresh scintillating screens, not affected by irreversible damage effects. For comparison, the calibration values from the work of Buck et $a l .{ }^{20}$ are listed for two screens also investigated in the earlier work.

\begin{tabular}{lccc}
\hline \hline Screen & $\begin{array}{c}\text { Absolute fluorescence efficiency } \\
\left(10^{9} \mathrm{ph} / \mathrm{sr} / \mathrm{pC}\right)\end{array}$ & $\begin{array}{c}\text { Saturation threshold } \\
\left(10^{3} \mathrm{pC} / \mathrm{mm}^{2}\right)\end{array}$ & $\begin{array}{c}\text { Birks' constant } \\
\left(10^{-5} \mathrm{~mm}^{2} / \mathrm{pC}\right)\end{array}$ \\
\hline Kodak BioMAX MS & $7.6 \pm 1.3$ & $1.6 \pm 0.2$ & $7.1 \pm 0.8$ \\
Cawo OG BACK & $5.8 \pm 1.0$ & $1.8 \pm 0.2$ & $6.0 \pm 0.6$ \\
Cawo OG FRONT & $3.7 \pm 0.7$ & $1.8 \pm 0.2$ & $6.1 \pm 0.7$ \\
Konica Minolta OG 400 & $3.7 \pm 0.7$ & $1.9 \pm 0.2$ & $5.8 \pm 0.6$ \\
Carestream Lanex Regular & $3.1 \pm 0.6$ & $3.5 \pm 0.3$ & $3.9 \pm 0.6$ \\
Kodak Lanex Fine & $1.0 \pm 0.2$ & & \\
\hline Kodak BioMAX MS (Buck et al. $)$ & $14.8 \pm 1.3$ & & \\
Kodak Lanex Fine (Buck et al. $)$ & $1.75 \pm 0.15$ & & \\
\hline \hline
\end{tabular}

TABLE II. List of typical input parameters for the absolute calibration.

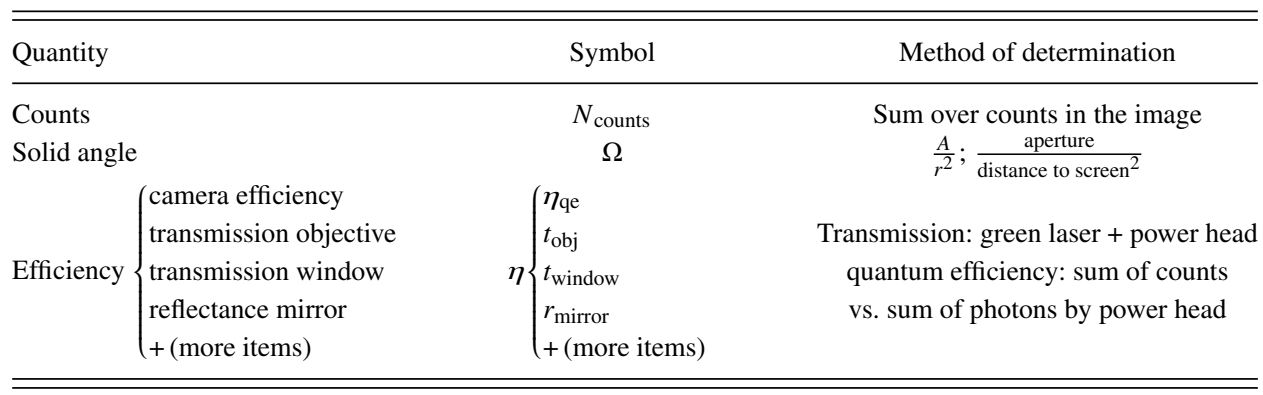


Lanex Fine of $(1.05 \pm 0.09) \times 10^{9} \mathrm{ph} / \mathrm{sr} / \mathrm{pC}$ confirming the current calibration value of $(1.0 \pm 0.2) \times 10^{9} \mathrm{ph} / \mathrm{sr} / \mathrm{pC}$ within the measurement uncertainty (see detailed derivation in the supplementary material). Consequently, the charge in LPA experiments has likely been underestimated in the past when the original calibration data of Buck et al. ${ }^{20}$ were applied.

Moreover, we have investigated the responses of screens from an asymmetric cassette, e.g., Cawo OG F/B. For the original $\mathrm{x}$-ray imaging application, scintillating screens are used to irradiate an analog film that is not sensitive to x-rays. In order to enhance the sensitivity, the scintillating screens are arranged in a sandwich structure (screen-film-screen). Although most cassettes use two identical screens, some, e.g., Cawo OG F/B are produced in a "front" and a "back" version, which are generally delivered in a single package. We found that the Cawo OG "back-side" screen has an efficiency that is $50 \%$ higher than the efficiency of the "front-side" screen.

\section{B. Saturation effects}

Beyond the linear region of the calibration curves, the photon-to-charge conversion efficiency is reduced due to saturation in the active layer of the scintillator. Birks' law, ${ }^{26}$ describing the saturation of scintillators, is used to fit the response curve of the scintillator,

$$
\rho_{\text {scint }}=\frac{\rho_{\text {ICT }}}{1+B \rho_{\text {ICT }}},
$$

where the fit parameter B is Birks' constant. Here, $\rho_{\mathrm{ICT}}$ is the applied peak charge density which is determined by the electron bunch charge measured by the ICT and the beam profile of the scintillator in the linear region. Assuming a constant beam shape, we calculated $\rho_{\text {ICT }}$ in the saturated regime using the charge information given by the ICT. $\rho_{\text {scint }}$ is the peak charge density measured on the scintillator. The saturation threshold value $\rho_{\text {sat }}$ is defined as the peak charge density, at which the scintillation signal has dropped to $90 \%$ compared to the linear behavior. This arbitrary measure is chosen such that the saturation effect can be clearly distinguished from relative measurement uncertainties in the linear case. Figure 4 shows a saturation curve of Kodak BioMAX MS with increasing electron peak charge density. The dashed line shows the linear correlation of $\rho_{\text {scint }}$ and $\rho_{\mathrm{ICT}}$, while the solid curve indicates the fit along the measured data. The resulting threshold values and the fit parameter B for the different screens are shown in Table I. It should be noted that the experimental implementation of the setup potentially underestimates this effect. For the highest applied charges, the duration of the pulse train is in the order of $100 \mu \mathrm{s}$ and becomes comparable to the lifetime of the excited state. Thus, electron bunches in the tail of the bunch train have an enhanced probability to re-excite atoms that have already relaxed back and thus add less to saturation. This effect has been included in Fig. 4 as an increased uncertainty toward lower scintillation peak charge density and is only relevant for the last two data points.

At high peak charge densities, all investigated scintillating screens start to saturate. A weakly focused electron beam was used to increase the peak charge density by more than two

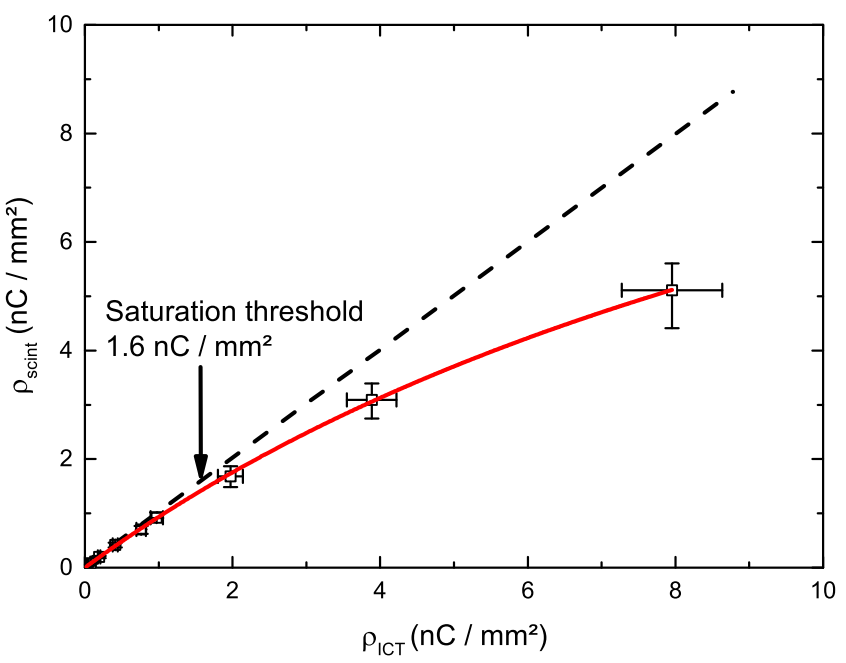

FIG. 4. Response function of Kodak Biomax MS showing saturation: The peak charge density measured by the screen vs. the peak charge density calculated from the beam profile of the scintillator and the charge information given by the ICT. The bunch profile shows a significant saturation toward higher charges. The measured data are fitted with Birks' law of saturation [solid line, see Eq. (2)]. The black dashed line indicates $\rho_{\text {scint }}=\rho_{\text {ICT }}$.

orders of magnitude compared to previous saturation studies..$^{20}$ In contrast to Ref. 20, we observe saturation of the scintillator starting when applying peak charge density on the order of $\mathrm{nC} / \mathrm{mm}^{2}$.

\section{Degradation effects}

Besides reversible saturation, additional and previously unreported beam-induced degradation occurs. In order to separate saturation from degradation damage, reference measurements were performed with a low charge of $60 \mathrm{pC}$ after each increment of the bunch charge to get a reasonable estimate for the degeneration caused by the increasing charge densities applied during the calibration measurements. Typically, it took several minutes to switch to the reference measurement. The beam-induced fluorescence reduction is defined as the reduction of the fluorescence signal after a recovery time of several minutes. Due to a limited access time to the accelerator, the recovery behavior of the screens could not be studied at longer time scales. Nevertheless this effect becomes relevant when frequently irradiating the scintillating screens with relativistic electrons, i.e., LPA experiments performed with Hz-repetition rate for several hours (see Fig. 5). After applying the cumulative charge of the entire calibration curve, the beam-induced damage by the measurement itself is consistently circa $15 \%$ for all screens except Kodak Lanex Fine, which did not show any decay. Again, this effect becomes relevant only at high electron doses, i.e., the last 2-3 data points in Fig. 4. The values in Table I (third and fourth columns) and Fig. 4 are corrected for this damage by adding the reduction caused by the measurement to the measured signal.

For a reliable bunch charge diagnostic, the long-term stability and possible degradation of the scintillating screens has to be quantified. Already the calibration curves in Fig. 3 include some non-reversible degradation with accumulated dose confirmed by intermediate low-charge reference shots. In order 


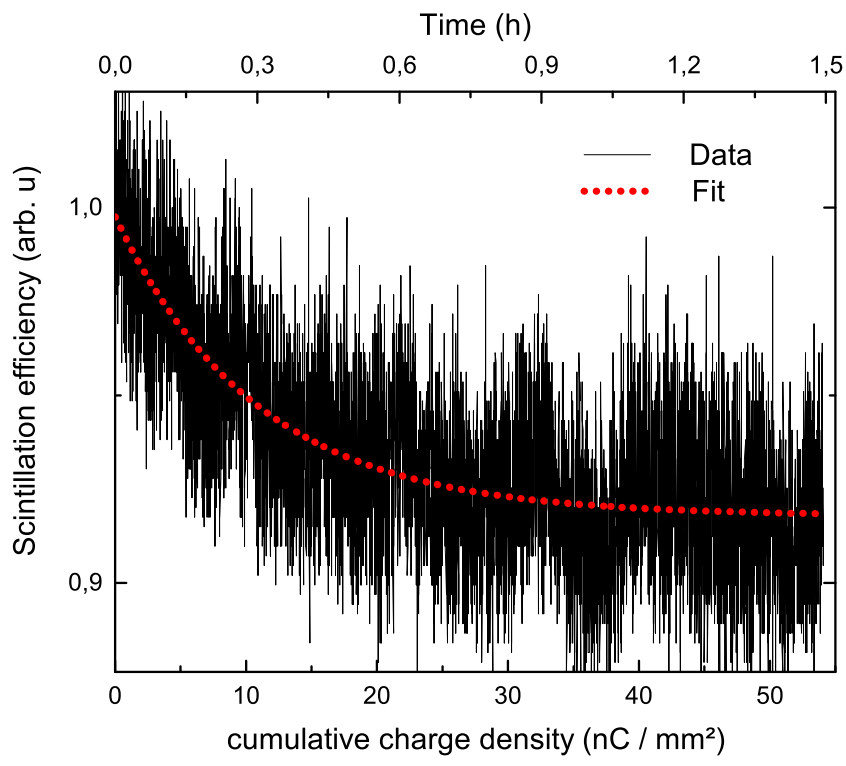

FIG. 5. Long-term performance test with Konica Minolta: The screen was irradiated for $1.5 \mathrm{~h}$ with $1 \mathrm{~Hz}$ repetition rate, $100 \mathrm{pC}$ charge, and a spot size of $2 \mathrm{~mm}^{2}$ at FWHM. The data were fitted with an exponential decay function that has an offset value at approximately 0.9 . The decay of the photon signal during this experiment was $9 \%$.

to further investigate this apparent beam-induced degradation, we conducted two dedicated long-term irradiation tests. These tests were independent from the calibration measurements and served as a different method than the above mentioned one to study irreversible damage effects in a much more controlled way. For each one, we used a fresh sample of one specific type of scintillating screen, i.e., Konica Minolta. These tests were performed with a constant charge density per shot far below the saturation threshold to exclude reversible saturation during the long-term tests. The electron beam parameters were chosen to represent LPA conditions as close as possible. Every second, the screen was irradiated by an electron bunch with a charge of $100 \mathrm{pC}$ over a duration of $90 \mathrm{~min}$. The FWHM bunch area was kept at $2 \mathrm{~mm}^{2}$ yielding electron densities at the target on the order of $9 \mathrm{pC} / \mathrm{mm}^{2}$. In the following, the results from two runs with almost equal beam parameters are described. Figure 5 shows the fluorescence signal as a function of the applied cumulated electron charge density over time. The data were fitted with an exponential decay function with an offset at around 0.9 to determine the loss of scintillation efficiency. A significant drop of $9 \%$ in the emitted scintillation efficiency over $1.5 \mathrm{~h}$ was observed. In such a case, the charge will be underestimated accordingly. The influence of the repetition rate and the charge density on the long-term stability, i.e., the decrease of the scintillation signal per total electron dose, was not investigated and should be studied in future work.

Figure 6 displays the temporal evolution of the efficiency during a second long-term test, performed under similar conditions as in the first run. First, the scintillator also shows a decay as observed in Fig. 5. The beam profile for a representative shot onto the scintillator at an integrated dose of $50 \mathrm{nC} / \mathrm{mm}^{2}$ is illustrated in Fig. 6. At a cumulative charge density of around $52 \mathrm{nC} / \mathrm{mm}^{2}$, the response function shows a sharp increased peak at which the screen lights up brightly with a hole in its center. Afterwards the screen is permanently damaged. Due to

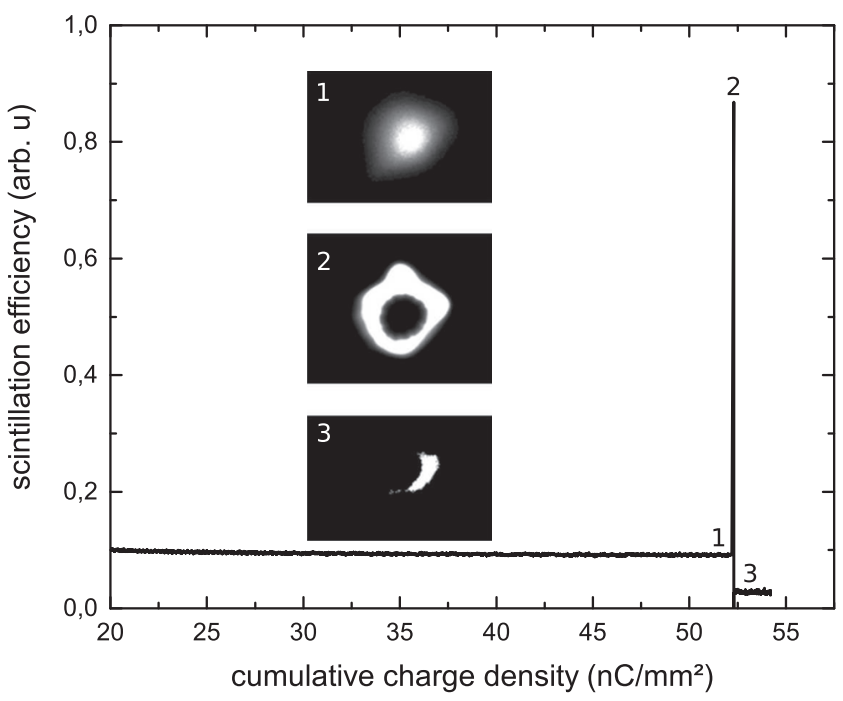

FIG. 6. Damage of Konica Minolta during long-term test: The data were taken at a different run with equal parameters as presented in Fig. 5. After applying a cumulative dose of $52 \mathrm{nC} / \mathrm{mm}^{2}$, the screen shows a bright peak and is permanently damaged afterwards.

the long irradiation time needed to cause this effect, we could not investigate how reproducible this sudden damage is, but it is worth recording its occurrence.

\section{IMPLEMENTATION OF THE LANEX CALIBRATION AT EXTERNAL LABORATORIES}

Here we explain how potential users can implement the presented calibration results to measure the charge of relativistic electron bunches.

The values in Table I give the absolute light output into a small solid angle around the forward direction as a function of incident charge. In principle, these values could be used to directly obtain charge figures from absolutely measured photon numbers. The problem with this brute force approach is that it requires a precise knowledge of the absolute transmission of the imaging system and the camera response. The total photons emitted by the scintillator per steradian $N_{\text {Total }}$ can be determined as

$$
N_{\text {Total }}=\frac{N_{\text {Counts }}}{\Omega \eta},
$$

where $N_{\text {Counts }}$ is the absolute amount of counts detected by the CCD-camera and $\Omega$ denotes the collection angle. The efficiency of the specific optical detection system $\eta$ is given by

$$
\eta=\eta_{\mathrm{qe}} \cdot t_{\mathrm{obj}} \cdot t_{\text {window }} \cdot r_{\text {mirror }} .
$$

Hereby, $\eta_{\mathrm{qe}}$ is the camera efficiency, $t_{\mathrm{obj}}$ and $t_{\text {window }}$ symbolize the transmission value through the objective and the vacuum window, respectively, and $r_{\text {mirror }}$ is the reflectivity of the turning mirror. Whereas $N_{\text {Counts }}$ and $\Omega$ are easy to determine, $\eta_{\text {qe }}$ is rather complex. Each element (e.g., ND filter, bandpass filter, mirror, vacuum window, camera objective, etc.) of the optical detection system has to be calibrated; i.e., the spectral transmission has to be multiplied with the spectrum of the scintillator, which is similar for all screen types (an example spectrum of Kodak BioMax MS is given in the 


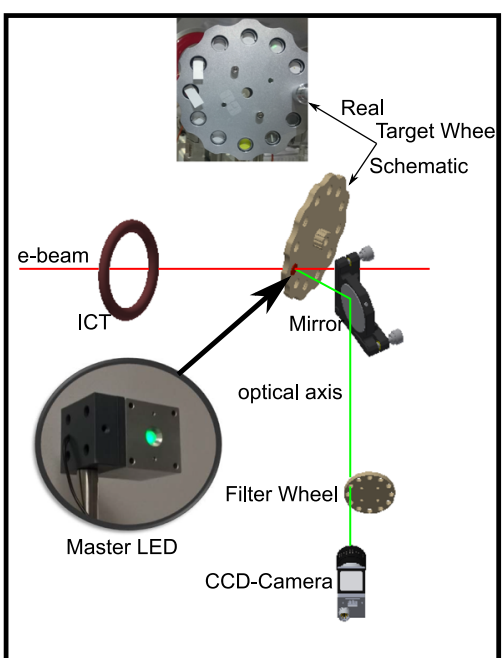

Step 1: Calibration experiment

+ cross calibration to master LED

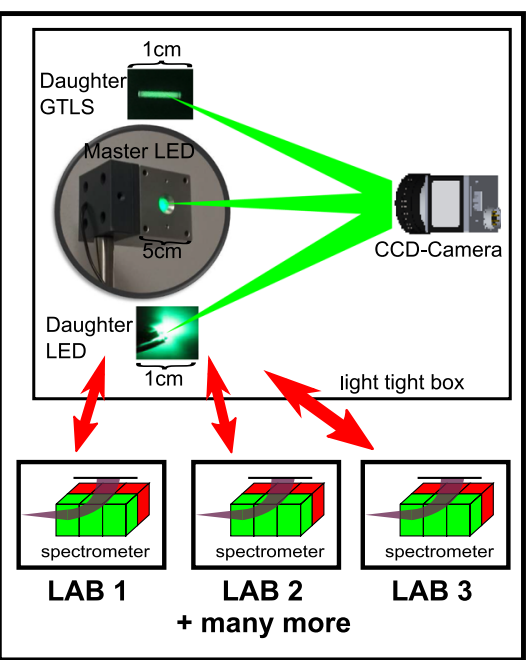

Step 2: Cross calibration of daughter light sources to master LED + distribution to other LABS
FIG. 7. Schematic overview for the implementation of the calibration in the electron diagnostic in an external LPA. Step 1 is the calibration of the scintillation screens and the cross calibration to a master LED in a RF-accelerator facility as described in this article (see also Fig. 1). Second step is the cross calibration of daughter light source, e.g., a GTLS or a small and vacuum compatible LED. Subsequently, these calibrated daughter sources can be implemented in any laboratory. supplementary material). Special care is needed for the calibration of the camera, as the efficiency of the CCD camera (photon-to-count) is not equal to the quantum efficiency (probability for an incident photon to create an electron-hole pair) of the CCD chip but also depends on the transformation ratio from the analog CCD signal to the amount of digital counts. This approach has the clear disadvantage that every change in the setup and the optical detection system directly affects the charge determination. Therefore a precise knowledge of each parameter in Eq. (3) is required. Additionally, the error in determining the signal of the screen (photons/sr) adds to the calibration error reported in Table I.

These problems can be overcome by calibrating the optical detection system with a constant brightness reference light source (called master light source, MLS in the following). This light source is directly compared with the signal of the scintillating screen through the same optical system as during the LINAC-based screen calibration procedure. By applying the MLS specific charge density $Q / A_{\mathrm{MLS}}$ and taking into account the measured ratio between the screen $B_{\text {screen }}$ and the MLS brightness $B_{\mathrm{MLS}}$ an to be measured charge density $Q / A$ can be expressed as

$$
Q / A=\frac{B_{\text {screen }}}{B_{\mathrm{MLS}}} Q / A_{\mathrm{MLS}},
$$

regardless of the imaging system. $Q / A_{\mathrm{MLS}}$ depends on the master light source and was obtained during the calibration campaign. However, due to its rather large geometrical dimensions and the vacuum incompatibility, the source is not implemented directly. Instead, it is used to cross-calibrate any amount of daughter light sources (DLSs) in a standalone setup (see the right image in Fig. 7) for distributing the calibration onto different experiments, where such a DLS is placed at the position of or next to the scintillating screen. A charge density $Q / A$ can then be determined via

$$
Q / A=\frac{B_{\text {screen }}}{B_{\mathrm{MLS}}} \frac{B_{\mathrm{MLS}}}{B_{\mathrm{DLS}}} Q / A_{\mathrm{MLS}},
$$

where $B_{\mathrm{MLS}} / B_{\mathrm{DLS}}$ is the cross-calibration factor between the MLS and the DLS.
In the past, calibrated gaseous tritium light sources (GTLSs) were used as MLSs/DLSs due to their small size and supposedly well-predictable light output. However, their main drawback beyond a certain radiation hazard is an aging behaviour that is not synchronous with the well-known tritium decay rate but also depends on an unknown degradation of their luminous phosphor. This makes it impossible to confidently predict and correct for the degradation of the light output over time. Therefore, for the use as MLS, the tritium capsules would have to be calibrated in a LINAC beamtime typically every year. The obvious solution for this problem is to design a truly constant MLS that is by far more accessible than a LINAC beamtime. Such a source can be found at the Helmholtz-Zentrum Dresden-Rossendorf ${ }^{28}$ or at the Ludwig-Maximilians-Universität München.

Finally, this method eliminates most error sources and enables the transport of calibration results to other laboratories. The new LED-based MLS is an improvement over the previously reported $\mathrm{MLS}^{20}$ because the amount of emitted light is stable over time. In certain scenarios, GTLSs, implemented as DLSs, are still preferred due to their small size and vacuum compatibility. The overall error of the charge density determination using a calibrated DLS will be in the order of $10 \%$.

\section{CONCLUSION}

We have presented absolute charge calibration measurements, saturation effect study, and long-term stability tests for various scintillating screens that are commonly used as the diagnostic for short-pulsed electron bunches with low average current and spread over a rather large area as in the case of LPAs. The measurements were performed with sub $10 \mathrm{ps}, 23.5 \mathrm{MeV}$ electron beams from the ELBE linear accelerator at the Helmholtz-Zentrum Dresden-Rossendorf. The absolute scintillation efficiency of the different scintillation screens was measured to vary over almost one order of magnitude. Kodak BioMAX MS shows the brightest photon response with an absolute fluorescence efficiency of 
$(7.6 \pm 1.3) \times 10^{9}$ photons/sr/pC but also is the screen which saturates first. For comparison, we have calculated the absolute response of Kodak Lanex Fine based on the experimental data provided by Glinec et al. ${ }^{19}$ The resulting value of $(1.05 \pm 0.09) \times 10^{9} \mathrm{ph} / \mathrm{sr} / \mathrm{pC}$ agrees to our value of $(1.0 \pm 0.2) \times 10^{9} \mathrm{ph} / \mathrm{sr} / \mathrm{pC}$ within the measurement uncertainties.

A saturation effect was visible when applying peak charge densities in the order of $\mathrm{nC} / \mathrm{mm}^{2}$. This is about three orders of magnitude higher than charge densities reached in current LPA experiments and can therefore generally be neglected when analyzing the spectrometer images.

Finally the long-term stability for a selected type of screen, i.e., Konica Minolta, was tested. We show that a typical electron dose, i.e., $9 \mathrm{pC} / \mathrm{mm}^{2}$, applied for $1.5 \mathrm{~h}$ at $1 \mathrm{~Hz}$ leads to a significant decrease of the fluorescence efficiency. Additionally, we found that heat damage of LANEX screens becomes an issue after prolonged continuous use. Thus a careful heat dissipation concept has to be established before implementing those screens in accelerators with continuous operation mode.

The absolute calibration factors given in this work have the disadvantage that they require a precise calibration of the screen imaging system's spectrally and spatially dependent throughput. In order to facilitate the calibration, we offer a relative screen calibration based on absolutely calibrated reference daughter light sources. Since the GTLS luminosity is not stable over time, a new concept for the cross-calibration of the scintillating screens was developed. The MLS/DLS approach simplifies the application and transportation of this calibration study significantly. Additionally, the uncertainty of the charge determination across laboratories can be minimized below $10 \%$ if a carefully calibrated DLS is implemented.

\section{SUPPLEMENTARY MATERIAL}

See supplementary material for the calculation of the reference points in Fig. 3 and the spectrum of Kodak BioMAX MS.

\section{ACKNOWLEDGMENTS}

This work was supported by EC Horizon 2020 LASERLAB-EUROPE/LEPP (Contract No. 654148), DFG through Munich Center of Advanced Photonics (Exc 158) and SFB Transregio TR18 funding schemes, EU through H2020 project Euratom (Contract No. 633053), and MPG through International Max-Planck Research School of Advanced Photon Science (IMPRS-APS), the Helmholtz Association ARD research topic, and the Virtual Institute VH-VI-503.

${ }^{1}$ T. Tajima and J. M. Dawson, Phys. Rev. Lett. 43, 267 (1979).

${ }^{2}$ E. Esarey, C. B. Schroeder, and W. P. Leemans, Rev. Mod. Phys. 81, 1229 (2009).

${ }^{3}$ M. C. Downer, R. Zgadzaj, A. Debus, U. Schramm, and M. C. Kaluza, Rev. Mod. Phys. 90, 035002 (2018).

${ }^{4}$ E. W. Gaul, M. Martinez, J. Blakeney, A. Jochmann, M. Ringuette, D. Hammond, T. Borger, R. Escamilla, S. Douglas, W. Henderson, G. Dyer, A. Erlandson, R. Cross, J. Caird, C. Ebbers, and T. Ditmire, Appl. Opt. 49, 1676 (2010).

${ }^{5}$ U. Schramm, M. Bussmann, A. Irman, M. Siebold, K. Zeil, D. Albach, C. Bernert, S. Bock, F. Brack, J. Branco, J. Couperus, T. Cowan, A. Debus, C. Eisenmann, M. Garten, R. Gebhardt, S. Grams, U. Helbig, A. Huebl,
T. Kluge, A. Köhler, J. Krämer, S. Kraft, F. Kroll, M. Kuntzsch, U. Lehnert, M. Loeser, J. Metzkes, P. Michel, L. Obst, R. Pausch, M. Rehwald, R. Sauerbrey, H. Schlenvoigt, K. Steiniger, and O. Zarini, J. Phys.: Conf. Ser. 874, 012028 (2017).

${ }^{6}$ C. G. R. Geddes, C. S. Toth, J. Van Tilborg, E. Esarey, C. B. Schroeder, D. Bruhwiler, C. Nieter, J. Cary, and W. P. Leemans, Nature 431, 538 (2004).

${ }^{7}$ J. Faure, Y. Glinec, A. Pukhov, S. Kiselev, S. Gordienko, E. Lefebvre, J.-P. Rousseau, F. Burgy, and V. Malka, Nature 431, 541 (2004).

${ }^{8}$ S. P. D. Mangles, C. D. Murphy, Z. Najmudin, A. G. R. Thomas, J. L. Collier, A. E. Dangor, E. J. Divall, P. S. Foster, J. G. Gallacher, C. J. Hooker, D. A. Jaroszynski, A. J. Langley, W. B. Mori, P. A. Norreys, F. S. Tsung, R. Viskup, B. R. Walton, and K. Krushelnick, Nature 431, 535 (2004).

${ }^{9}$ C. B. Schroeder, C. Tóth, B. Nagler, A. J. Gonsalves, K. Nakamura, C. G. R. Geddes, E. Esarey, S. M. Hookert, and W. P. Leemans, in Conference Proceedings_Lasers and Electro-Optics Society Annual Meeting (IEEE, 2007) Vol. 2, p. 538.

${ }^{10}$ X. Wang, R. Zgadzaj, N. Fazel, Z. Li, S. A. Yi, X. Zhang, W. Henderson, Y.-Y. Chang, R. Korzekwa, H.-E. Tsai, C.-H. Pai, H. Quevedo, G. Dyer, E. Gaul, M. Martinez, A. C. Bernstein, T. Borger, M. Spinks, M. Donovan, V. Khudik, G. Shvets, T. Ditmire, and M. C. Downer, Nat. Commun. 4, 1988 (2013).

${ }^{11}$ W. P. Leemans, A. J. Gonsalves, H. S. Mao, K. Nakamura, C. Benedetti, C. B. Schroeder, C. Tóth, J. Daniels, D. E. Mittelberger, S. S. Bulanov, J. L. Vay, C. G. R. Geddes, and E. Esarey, Phys. Rev. Lett. 113, 245002 (2014).

${ }^{12}$ J. P. Couperus, R. Pausch, A. Köhler, O. Zarini, J. M. Krämer, M. Garten, A. Huebl, R. Gebhardt, U. Helbig, S. Bock, K. Zeil, A. Debus, M. Bussmann, U. Schramm, and A. Irman, Nat. Commun. 8, 487 (2017).

${ }^{13}$ O. Lundh, J. Lim, C. Rechatin, L. Ammoura, A. Ben-Ismaïl, X. Davoine, G. Gallot, J.-P. Goddet, E. Lefebvre, V. Malka, and J. Faure, Nat. Phys. 7, 219 (2011).

${ }^{14}$ R. Morlotti, M. Nikl, M. Piazza, and C. Boragno, J. Lumin. 72-74, 772 (1997).

${ }^{15}$ K. A. Tanaka, T. Yabuuchi, T. Sato, R. Kodama, Y. Kitagawa, T. Takahashi, T. Ikeda, Y. Honda, and S. Okuda, Rev. Sci. Instrum. 76, 013507 (2005).

${ }^{16}$ S. Masuda, E. Miura, K. Koyama, and S. Kato, Rev. Sci. Instrum. 79, 083301 (2008).

${ }^{17}$ K. Zeil, S. D. Kraft, A. Jochmann, F. Kroll, W. Jahr, U. Schramm, L. Karsch, J. Pawelke, B. Hidding, and G. Pretzler, Rev. Sci. Instrum. 81, 013307 (2010).

${ }^{18}$ T. Bonnet, M. Comet, D. Denis-Petit, F. Gobet, F. Hannachi, M. Tarisien, M. Versteegen, and M. M. Aleonard, Rev. Sci. Instrum. 84, 013508 (2013).

${ }^{19}$ Y. Glinec, J. Faure, A. Guemnie-Tafo, V. M. Monard, J. P. Larbre, V. De Waele, J. L. Marignier, M. Mostafavi, V. Malka, and H. Monard, Rev. Sci. Instrum. 77, 103301 (2006).

${ }^{20}$ A. Buck, K. Zeil, A. Popp, K. Schmid, A. Jochmann, S. D. Kraft, B. Hidding, T. Kudyakov, C. M. S. Sears, L. Veisz, S. Karsch, J. Pawelke, R. Sauerbrey, T. Cowan, F. Krausz, and U. Schramm, Rev. Sci. Instrum. 81, 033301 (2010).

${ }^{21}$ K. Nakamura, A. J. Gonsalves, C. Lin, A. Smith, D. Rodgers, R. Donahue, W. Byrne, and W. P. Leemans, Phys. Rev. Spec. Top.-Accel. Beams 14, 062801 (2011).

${ }^{22}$ B. Hidding, G. Pretzler, M. Clever, F. Brandl, F. Zamponi, A. Lübcke, T. Kämpfer, I. Uschmann, E. Förster, U. Schramm, R. Sauerbrey, E. Kroupp, L. Veisz, K. Schmid, S. Benavides, and S. Karsch, Rev. Sci. Instrum. 78, 083301 (2007).

${ }^{23}$ Y. C. Wu, B. Zhu, K. G. Dong, Y. H. Yan, and Y. Q. Gu, Rev. Sci. Instrum. 83, 026101 (2012).

${ }^{24}$ G. E. Giakoumakis and D. M. Miliotis, Phys. Med. Biol. 30, 21 (1985).

${ }^{25}$ B. Yang, "A design report for the optical transition radiation imager for the LCLS undulator," Technical Report LCLS-TN-05-21 (SLAC, Stanford, 2005).

${ }^{26}$ J. B. Birks, D. Fry, L. Costrell, and K. Kandiah, The Theory and Practice of Scintillation Counting (Pergamon Press, Oxford, 1964).

${ }^{27}$ We have irradiated the scintillation screens afterwards with a broadband $\mathrm{X}$-ray source to test the homogeneity of the investigated screen samples. We have not measured any significant variation along the screens. Additionally, a fresh screen area was taken for each of the three campaigns to compare the absolute fluorescence efficiency resulting in very similar results.

${ }^{28} \mathrm{~A}$ cross-calibration measurement for daughter light sources can be performed by the authors including this work into the reference list of relevant publications making use of the calibration. The MLS is based on a constant current source driving a green LED with a specified lifetime of more than $10000 \mathrm{~h}$. Due to its infrequent use, this translates to a practical usability over several decades. 